\title{
NUEVOS DATOS SOBRE LOS PAMPHAGIDAE (ORTHOPTERA, CAELIFERA) DE LA PENÍNSULA IBÉRICA I. NUEVA SUBESPECIE DE EUMIGUS BOLÍVAR, 1878 DE LA SIERRA DE ALCARAZ (ALBACETE, ESPAÑA) ${ }^{1}$
}

\author{
J. J. Presa (*), V. Llorente $(* *)$ y M. D. García (*)
}

\begin{abstract}
RESUMEN
Se describe una nueva subespecie de Pamphagidae, Eumigus punctatus calarensis, de la Sierra de Alcaraz (Albacete, España), discutiéndose sus características diferenciales. Se presentan los datos sobre la producción de sonido y las manifestaciones acústicas. Se aportan nuevos datos sobre otras especies de Pamphagidae; entre ellos se discute la situación taxonómica de Acinipe mabillei, proponiéndose su consideración como especie válida, y se presentan los primeros datos conocidos sobre producción de sonido por el método del batido de tegminas (flapping) en panfágidos ibéricos, en Eumigus punctatus punctatus. Se designan lectotipo y paralectotipo de Kurtharzia nugatoria.

Palabras clave: Eumigus punctatus calarensis n. ssp., producción de sonido, Acinipe mabillei especie válida, nuevos datos sobre Pamphagidae.
\end{abstract}

\begin{abstract}
New data on Phamphagidae (Orthoptera, Caelifera) of the Iberian Peninsule I. New subpecies of Eumigus Bolívar, 1878 from the Sierra de Alcaraz (Albacete, Spain)

Eumigus punctatus calarensis, a new subespecies of Pamphagidae, is described from Sierra de Alcaraz, in Albacete province (Spain), and its characteristics are discussed. We present data about its sound production and acoustic signals. New data on other species of Pamphagidae are given; including discussion of the taxonomic status of Acinipe mabillei, its account as valid species is proposed. We present the first known data on sound production by flapping method in Iberian Pamphagidae, for Eumigus punctatus punctatus. Lectotype and paralectotype of Kurtharzia nugatoria are designated.
\end{abstract}

Key words: Eumigus punctatus calarensis n. ssp., sound production, Acinipe mabillei as valid species, new data on Pamphagidae.

\section{Introducción}

Desde la entrada en imprenta en 1995 de la revisión de los panfágidos ibéricos (Llorente \& Presa,
1997) se han producido escasas aportaciones al conocimiento de estos ortópteros (Barranco et al., 1996; Hernández et al., 1998). Aprovechando la descripción de un nuevo taxón, queremos rectificar

* Área de Zoología. Departamento de Biología Animal. Facultad de Biología. Universidad de Murcia. Campus de Espinardo. 30100 Murcia. España.

** Departamento de Biodiversidad. Museo Nacional de Ciencias Naturales. CSIC. José Gutiérrez Abascal 2. 28006 Madrid. España.

1 Trabajo parcialmente financiado con el proyecto PB96-1104 A de la DGICYT. 
algunos errores que se deslizaron en la obra antes mencionada y añadir nuevos datos sobre alguna de las especies.

\section{Resultados}

Eumigus punctatus calarensis ssp. nov.

Material tíPICo: Holotipo. O", España: "El Calar del Mundo", Albacete, 18-IV-1995. R. Gómez, leg." "UZM. Col. J.J.Presa". Paratipos: Albacete: Sierra de Alcaraz, en el Calar del Mundo, 1.500 m, 4 ర $0^{7}$ y 3 우 ㅇ , 19-VI-1990 (R. Gómez); 2 $\sigma^{7} \sigma^{x}$ y 6 우 우, 18-IV-1995 (R. Gómez); en Fuente del Buitre, $10^{7}$ y 1 오, 2-VII-1988 (L. Pulido). El Calar del Mundo, $10^{\text {T }}$ y 1 O , 14-VI-1999 (M. D. García leg.); Calar del Mundo, $10^{\pi}$, 1 \& , 18-IV-1995 (R. Gómez); Los Torcos, S. Alcaraz, Calar del Mundo, 3 ○ $^{7}$, 2-VII-88 (L. Pulido leg.).

El Holotipo y los Paratipos $1 \sigma^{\prime \prime}$ y 1 우 del Calar del Mundo, del 19-VI-1990 y 1 O de Los Torcos, del 2-VII-1988 quedan depositados en el Museo Nacional de Ciencias Naturales; los demás ejemplares quedan depositados en la colección del Área de Zoología del Departamento de Biología Animal de la Universidad de Murcia.

DESCRIPCIÓN: Tegumento rugoso cubierto de puntos hundidos alternando con pequeños granos irregulares que, a veces, llegan a formar aristas. Antenas en el macho de 15 a 17 artejos, relativamente largas, sobrepasando el borde posterior de los paranotos. En la hembra tienen de 16 a 18 artejos, sobrepasando el surco posterior y a veces llegando al borde posterior de los paranotos. Vértex en el macho casi horizontal a la altura del ojo y con las quillas laterales elevadas, quilla occipital presente; en la hembra se presenta bastante inclinado.

Pronoto con la quilla surcada longitudinalmente, en el macho tectiforme, bastante alta y curvada, surco posterior con la incisión bien patente, siendo la metazona más de 1/4 de la longitud total del pronoto; en la hembra semejante, con la quilla más o menos alta, poco curvada y relativamente fina.Tubérculo prosternal bastante grueso, con la arista casi recta o bilobulada, con dos granos en la parte posterior que, a veces, forman una quilla a cada lado.

Tegminas lobiformes y oval-alargadas, siendo más de dos veces más largas que anchas, con el borde superior (in situ) más arqueado que el inferior. La banda blanca longitudinal bien manifiesta, ocupando casi el tercio de su anchura y llegando hasta el ápice. En el macho cubren el primer terguito abdominal y a veces llegan hasta la mitad del segundo. En las hembras no llegan al borde posterior del primer terguito o raras veces lo sobrepasan.
Abdomen del macho comprimido, con la quilla marcada y con un diente grueso y corto al final de cada terguito, siendo más prominentes en los 4-5 primeros. Apertura timpánica trapezoidal. Epiprocto oval redondeado con el surco longitudinal oscurecido en sus lados, angosto y profundo en la base, estrechándose en toda su longitud, siendo a menudo imperceptible del tercio al quinto apical. Cercos cónicos y esbeltos, más largos que anchos, bastante aplanados, con el ápice más o menos agudo. Placa subgenital aproximadamente igual de larga que los dos esternitos anteriores juntos, con una sutura más o menos sinuosa. En la hembra, el diente del final de cada terguito es relativamente corto y romo. Epiprocto estrechamente romboidal, con el ápice agudo, el surco longitudinal en la base ancho y aquillado, desapareciendo en el cuarto apical; surco transversal bien patente, estrecho y representando casi siempre como una línea muy fina y oscurecida que cruza el surco longitudinal aproximadamente en el medio. Cercos cónicos, casi tan largos como anchos, con el ápice bastante agudo. Placa subgenital grande, con puntos hundidos; borde posterior formando cinco lóbulos bien manifiestos, siendo el central triangular más largo y más estrecho (cerca de $1 \mathrm{~mm}$. en la base) que los cuatro restantes, que son siempre redondeados.

Complejo fálico como en la figura 1a; valvas del pene anchas en la base y con el borde exterior (ventral) ampliamente redondeado, con los ápices relativamente gruesos y muy cortos y, aunque también dirigidos hacia delante, poco curvados; cerca del tercio apical, hacia el lado externo, hay una fosita oval-alargada y profunda, el lado interno está elevado y lleva una hilera de diminutos dientes que alcanza casi los ápices de las valvas; además en la parte basal de la fosita se produce un estrechamiento o incisión; casi toda la superficie de las valvas, incluso debajo de los rami, está diseminada de pequeños granos, a veces terminados en espinitas, siendo más visibles en los extremos. Carece de protuberancias bajo los rami. Epifalo como en la figura 1b, ancho, con las áncoras separadas entre sí y con el borde posterior algo estrecho; las elevaciones espinosas poco globosas, relativamente planas y paralelas entre sí, con el surco entre ellas estrecho y poco profundo, cada una de las elevaciones lleva de 12 a 21 dientes, poco prominentes y finos, siempre con algunos más diminutos en el mismo surco.

Oviscapto con las valvas dorsales altas y robustas, con el borde inferior externo ensanchado y saliente, formando una especie de filo; valvas ventrales más pequeñas y vistas desde abajo con la quilla transversal relativamente alta y débilmente 


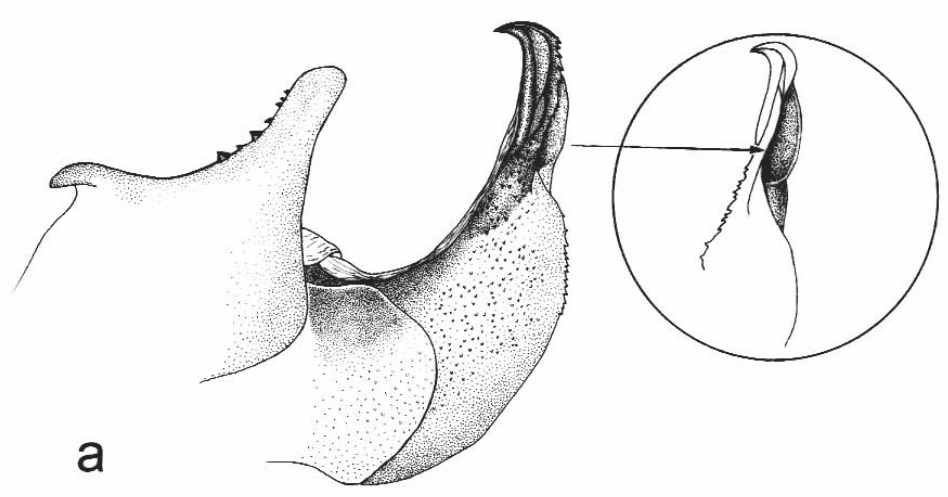

b

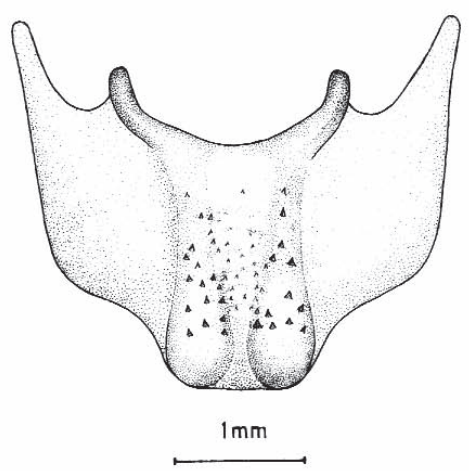

Fig. 1.-Eumigus punctatus calarensis ssp. n. a) complejo fálico en vista lateral; b) epifalo.

Fig. 1.-Eumigus punctatus calarensis ssp. n. a) phallic complex, lateral view; b) epiphallus. interna y dorsal, a menudo también la parte dorsal de los tarsos, rojo coralino, muy brillante en el macho y rojo violáceo, raras veces azul oscuro, en la hembra. El número de espinas, por lo general, es nueve en la cara externa y de ocho en la interna, además de la apical de cada lado.

Medidas en mm: Longitud del cuerpo: $\sigma^{7}, 33-35 ;$ o, $45-53$. Pronoto: $\sigma^{\pi}, 7,0-8,3 ;$; $, ~ 10,6-11,5$. Tegmina: $\sigma^{7}, 5,0: 2,2-7,0: 2,7$; \%, 6,5:2,9-9,0:3,4. Fémur posterior: o , $13,0: 3,6-14,2: 4,0 ;$; $, 16,8: 4,6-$ $19,0: 5,0$.

ETimología: Subespecie nominada en referencia a la Sierra del Calar del Mundo (Albacete) donde se han capturado tanto el holotipo como los paratipos.

Biología: Se han capturado ejemplares adultos desde abril hasta julio, a una altitud entre $1.500 \mathrm{y}$ $1.600 \mathrm{~m}$. El medio vegetal en que se encuentra corresponde a una comunidad pionera de crestas y laderas abruptas formada por caméfitos espinosos de porte almohadillado, con predominio fisiognómico de la genista Echinospartum boissieri, perteneciente a la Saturejo intrincatae - Echinospartetum boiossieri R.-G. \& R.-M. 1968.

DisCUSIÓN: Esta nueva subespecie oblicua, situada cerca del tercio basal, con el diente interno pronunciado; vistas del lado externo y ventral largas y estrechándose paulatinamente hacia el ápice.

Fémures posteriores con la quilla dorsal apenas aserrada; cara externa generalmente con manchas irregulares y puntos pardo oscuro sobre fondo pardo más o menos claro o amarillento; cara interna de color rosado intenso con las quillas superior e inferior formando un dibujo reticular en los $2 / 3$ basales y a veces dominando un tono más oscuro aquí y en los puntos y espinas situados en quillas superior e inferior; rodillas en ambos sexos con el lóbulo superior externo e interno, así como la base del inferior interno, pardo oscuro. Tibias del mismo par con la cara se diferencia claramente de las otras dos, E. punctatus punctatus (Bolívar, 1902) у E. punctatus templadoi Llorente y Presa, 1986, por la genitalia del macho, en especial por varios caracteres del epifalo y de las valvas del pene, que a continuación exponemos.

$$
\text { E. punctatus punctatus (Figuras 2c y 2d) }
$$

1.- Valvas del pene desde la base anchas con el borde externo (ventral) formando un ángulo recto redondeado; con los ápices relativamente cortos y curvados; fosita situada hacia el tercio apical de la valva (Fig. 2c)

2.- Epifalo con las áncoras bien separadas entre sí $(2,3$ $\mathrm{mm}$ ) y con el borde posterior ancho $(1,3 \mathrm{~mm})$, elevaciones espinosas globosas y casi paralelas entre sí, con el surco relativamente ancho y profundo; 

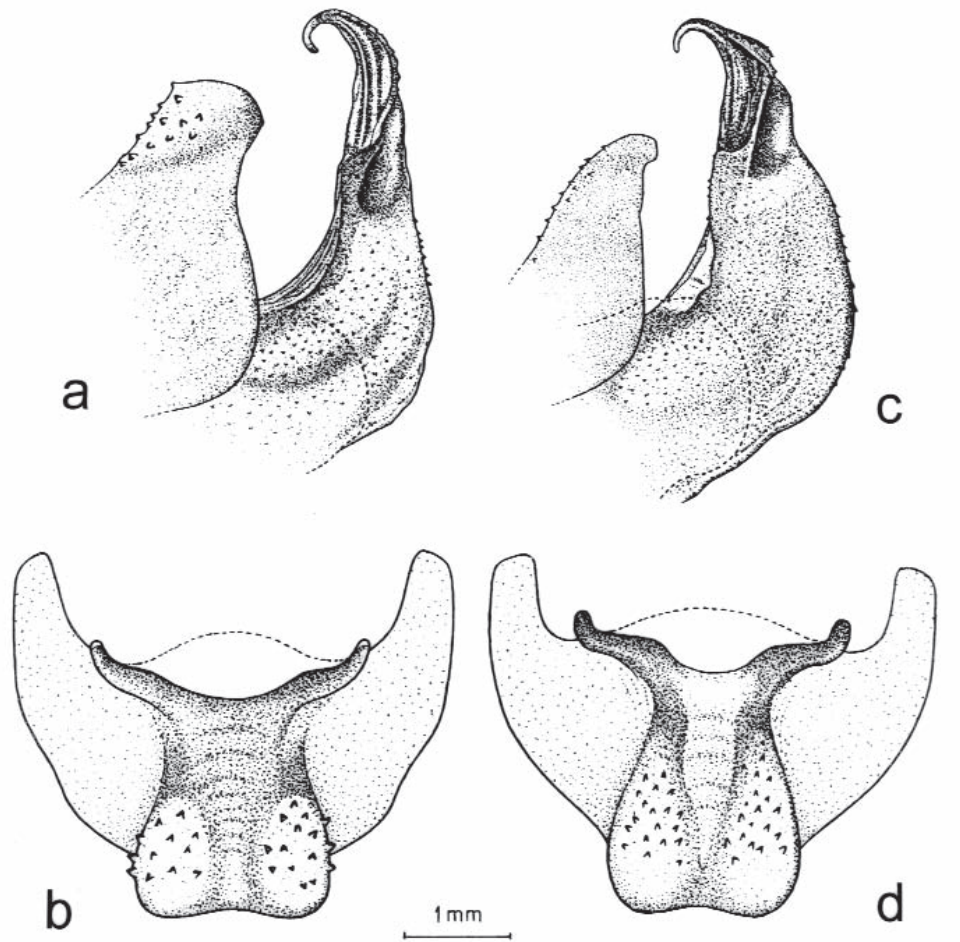

Fig. 2.-Eumigus punctatus templadoi: a) complejo fálico en vista lateral; b) epifalo. Eumigus punctatus punctatus: c) complejo fálico en vista lateral; d) epifalo.

Fig. 2.-Eumigus punctatus templadoi: a) phallic complex, lateral view; b) epiphallus. Eumigus punctatus punctatus: c) phallic complex, lateral view; d) epiphallus.

cada una de las elevaciones lleva de 16 a 25 dientes bastante gruesos formando un triángulo alargado (Fig. 2d).

\section{E. punctatus templadoi (Figuras 2a y 2b)}

1.- Valvas del pene desde la base más estrechas, con los ápices finos, largos y curvados; fosita situada más hacia el medio y más alejada del ápice (Fig. 2a).

2.- Epifalo con las áncoras muy separadas entre sí $(2,3$ $\mathrm{mm})$ y con el borde posterior ancho $(1,5 \mathrm{~mm})$; elevaciones espinosas muy globosas y claramente divergentes, con el surco más ancho que en las otras dos subespecies y de forma más irregular; cada una de las elevaciones lleva de 12 a 25 dientes más gruesos formando un círculo (Fig. 2b)

\section{E. punctatus calarensis ssp. nov.}

(Figuras $1 \mathrm{a}$ y $1 \mathrm{~b}$ )

1.- Valvas del pene desde la base anchas y con el borde externo (ventral) ampliamente redondeado; ápices muy cortos, gruesos y poco curvados; fosita más profunda y situada hacia el tercio apical de las valvas y más próxima al ápice (Fig. 1a).

2.- Epifalo más estrecho, con las áncoras casi un cuarto $(1,7 \mathrm{~mm})$ menos separadas entre sí y con el borde posterior alrededor de un tercio $(0,9$ $\mathrm{mm}$ ) más estrecho que en las otras dos subespecies; elevaciones espinosas del epifalo muy poco globosas, relativamente planas y paralelas entre sí, con el surco entre ellos más estrecho y muy poco profundo; cada una de las elevaciones lleva de 12 a 21 dientes menos prominentes y más finos que en los otros dos taxones y casi siempre con algunos diminutos en el mismo surco (Fig. 1b).

Producción DE SONIDO: Se han estudiado las manifestaciones acústicas de esta subespecie, producidas por la hembra en modo similar a lo observado en otros taxones de la familia Pamphagidae (García \& Presa, 1985; Clemente et al., 1990; Llorente et al., 1995; García et al., 1996) esto es, elevando para cantar las dos tegminas sincrónicamente, situándolas horizontales y golpeando con ellas las rodillas del segundo par de patas una vez levantadas éstas, produciendo un tamborileo claramente audible. En la subespecie estudiada, durante el canto, el extremo del abdomen queda ligeramente levantado y las antenas se disponen horizontalmente respecto al sustrato.

Se obtuvieron y estudiaron 23 cantos, producidos a lo largo del día 18 de junio de 1999, a partir de las 13 horas por una hembra recogida en Calar del Mundo (Albacete), 14-VI-1999 (M.D. García leg.). Fueron registrados, en el laboratorio del Área de Zoología de la Universidad de Murcia, con un magnetofón Uher 6000 y un micrófono Uher M-655, a una velocidad de grabación de $9,5 \mathrm{~cm} / \mathrm{seg}$., en el interior de un insectario de madera, provisto de vegetación, cuya fuente de luz y calor era una bombilla incandescente de $25 \mathrm{~W}$ de potencia. La temperatura en el interior del insectario era de $28-30^{\circ} \mathrm{C}$.

El sonido fue estudiado con el programa informático Avisoft ${ }^{\circledR}$ SAS Lab Pro 3.0 previa digitalización con una tarjeta Sound Blaster $\AA$ AWE64® Gold, con una frecuencia de muestreo de $44 \mathrm{kHz}$, y su tratamiento con un filtro Krohn-Hite 3550 a fin de evitar interferencias de baja frecuencia. Para 

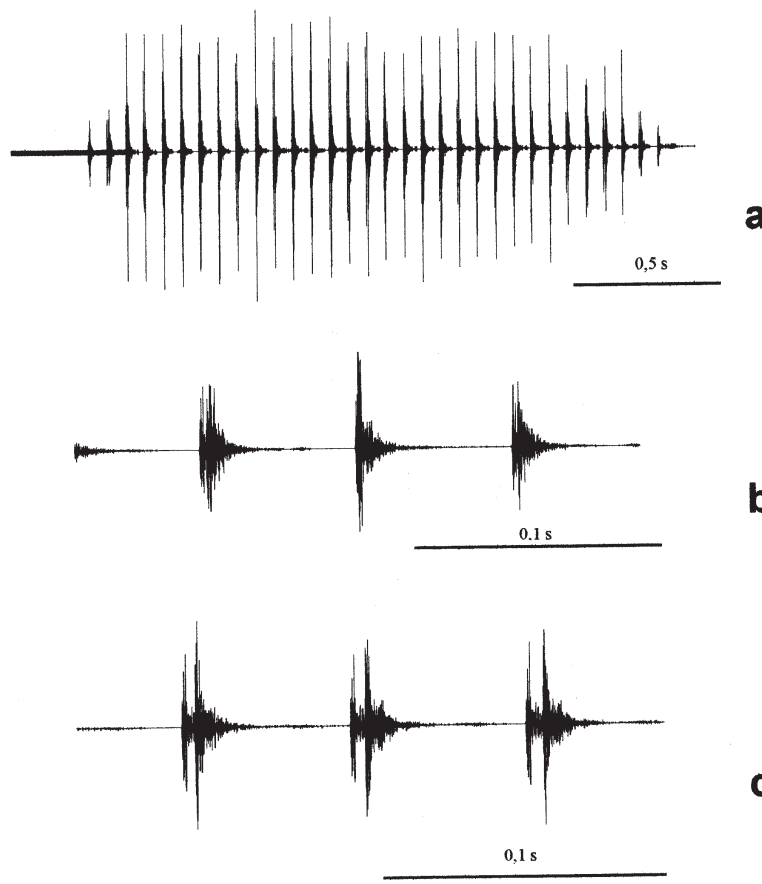

Fig. 3.- Manifestaciones acústicas de Eumigus punctatus calarensis ssp. n. a) equema completo; b) detalle de sílabas sincrónicas; c) detalle de sílabas asincrónicas.

Fig. 3.- Sound produced by Eumigus punctatus calarensis ssp. n. a) whole echeme; b) synchronous syllables; c) asynchronous syllables.

obtener los datos relativos a la frecuencia principal de emisión de la señal se aplicó el método de la transformada de Fourier (FFT), con una ventana de 256 puntos de amplitud.

El canto está constituido por equemas aislados (Fig. 3a) cuya duración media es de 1,896 segundos (máx. 2,040; mín. 1,754). Los equemas están compuestos por un número bastante constante de sílabas, 32 por término medio (máx. 34; mín. 30). La tasa de emisión silábica es de 16 ó 17 sílabas/segundo. Las sílabas, separadas entre sí por espacios de entre 0,035 a 0,040 segundos, tienen una duración de 0,020 segundos, aproximadamente, cuando se trata de sonidos sencillos, esto es, producidos de modo sincrónico por las dos tegminas (Fig. 3b). En ocasiones las tegminas producen sonido con un cierto desfase y la sílaba resultante tiene una duración superior, alrededor de 0,025 segundos (Fig. 3c).

Los cantos se emiten aisladamente, sin una cadencia temporal fija, pero su estructura es muy constante.

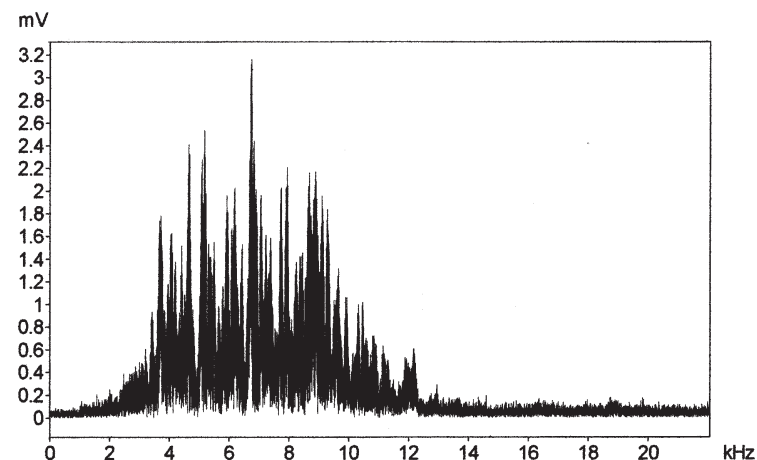

Fig. 4.- Espectro de frecuencia de emisión de las manifestaciones acústicas de Eumigus punctatus calarensis ssp. $\mathrm{n}$.

Fig. 4.- Main frequency spectrum of sound produced by Eumigus punctatus calarensis ssp. $\mathrm{n}$.

El sonido producido ocupa una banda de frecuencia amplia; el cuartil inferior $(25 \%$ de la energía) se sitúa alrededor de los $5 \mathrm{kHz}$; el cuartil intermedio (50\% de la energía) hacia los $7 \mathrm{kHz}$ y el cuartil superior (75\% de la energía) hacia los 9 ó 10 $\mathrm{kHz}$. Los picos principales de la emisión varían desde 5 hasta $8 \mathrm{kHz}$, aunque tienden a situarse entre 6 y 7 kHz. (Fig. 4)

El sonido de esta subespecie es similar, en su producción y sus características generales, al conocido de otras especies de la misma familia (Clemente et al., 1990; Llorente et al., 1995; García et al., 1996). Comparado con el canto de la subespecie E. puncatus templadoi se aprecian algunas diferencias; por ejemplo, la duración del canto de la nueva subespecie es menor y está formado por un número de sílabas notablemente más bajo; sin embargo, la duración unitaria de las sílabas es prácticamente el doble que en la otra subespecie, y están separadas por espacios más cortos. A pesar de estas diferencias, la tasa de emisión silábica es similar en los dos taxones. Los datos relativos al espectro de frecuencia de emisión son también similares a los conocidos para la otra subespecie (Tabla 1).

\section{Acinipe segurensis (Bolívar, 1908)}

Valencia debe ser excluida de su área de distribución, pues los ejemplares de dicha provincia pertenecen a A. deceptoria y así aparecen en la relación de material de esa especie. 
Tabla 1.- Resumen comparativo de las características métricas y físicas de los sonidos producidos, en cautividad, por las hembras de Eumigus punctatus templadoi y Eumigus punctatus calarensis n.ssp.

Table 1.- Comparative summary of metrical and physical characteristics of sounds produced, in captivity, by females of Eumigus punctatus templadoi and Eumigus punctatus calarensis n.ssp.

\begin{tabular}{lcccccc}
\hline Taxón & $\begin{array}{c}\text { Duración } \\
\text { equema (seg.) }\end{array}$ & $\begin{array}{c}\text { Número } \\
\text { sílabas }\end{array}$ & $\begin{array}{c}\text { Duración } \\
\text { sílabas (seg.) }\end{array}$ & $\begin{array}{c}\text { Intervalo entre } \\
\text { sílabas (seg.) }\end{array}$ & $\begin{array}{c}\text { Tasa emisión } \\
\text { (sílabas/seg) }\end{array}$ & $\begin{array}{c}\text { Frecuencia } \\
\text { principal (Hz) }\end{array}$ \\
\hline E. punctatus templadoi & $\begin{array}{c}2,810 \\
(1,360-3,650)\end{array}$ & $\begin{array}{c}47 \\
(36-59)\end{array}$ & 0,010 & 0,050 & $16-17$ & 7000 \\
E. punctatus calarensis n. ssp. & $\begin{array}{c}1,896 \\
(1,754-2,040)\end{array}$ & $\begin{array}{c}32 \\
(30-34)\end{array}$ & 0,020 & 0,035 & $16-17$ & 6000 \\
& & & & & 7000 \\
\hline
\end{tabular}

\section{Ocnerodes brunneri (Bolívar, 1876)}

En relación con esta especie queremos hacer un breve comentario acerca de la variabilidad morfológica externa de algunos ejemplares. Estos ejemplares, dos machos de Puertollano (Ciudad Real), un macho de Manzanares (Ciudad Real) (Ocnerodes fallaciosus Bol. det.) y un macho de Santa Elena (Jaén), por su apariencia externa y, en especial, por la estructura del tegumento, menos rugoso en todo el cuerpo y en los fémures posteriores, recuerdan a O. fallaciosus Bolívar, 1912. Este hecho llevó a Bolívar (1912) a considerar los ejemplares que él estudió como pertenecientes a esta última especie. Sin embargo, por el tubérculo prosternal están más próximos al grupo prosternalis; aunque tampoco tienen bien definidas las quillas laterales del tubérculo. Estos ejemplares, sobre la base de la estructura de la genitalia de los machos, fueron adjudicados a $O$. brunneri brunneri, los dos machos de Puertollano, y a $O$. brunneri cyanipes, el macho de Manzanares y el de Santa Elena (Jaén). Esto nos afirma en el valor identificador que le hemos dado a la genitalia.

\section{Acinipe mabillei (Bolívar, 1878)}

Recientemente, Heller et al. (1998: 16) proponen la desaparición de este binomio y su sustitución por el de Acinipe affinis (Fieber, 1853) apoyándose, en nuestra opinión, en una aplicación equivocada del principio de prioridad y en contra de la opinión generalizada, como aparece en Harz (1975), Presa \& García (1983), Otte (1994). A continuación exponemos los datos de que disponemos lo más brevemente posible, con objeto de aclarar este problema.
Pamphagus affinis fue descrita brevemente por Fieber de España. Bolívar (1876) da una descripción en castellano de la especie, indicando que no es posible decidir si pertenece al género Pamphagus Thunberg, 1815 o a Acocera Serville, 1838. Este mismo autor en 1878, en el mismo artículo en que describe Pamphagus mabillei, la considera como Pamphaginae inc. sedis, en ambos casos la considera de Hispania.

Brunner (1882) no hace referencia alguna, sin embargo, en el registro alfabético, menciona un affinis de la página 206 que, sin embargo, no aparece en el texto de dicha página, donde se estudian P. expansus Brunner, 1882; P. deceptorius Bolívar, 1878 y $P$. marmoratus Fischer, 1854.

Cazurro (1888) dice: "respecto a Pamphagus affinis Fieb. no es posible encontrar especie a la cual pueda referirse".

Hay que esperar hasta Kirby (1910), quien la considera como perteneciente al género Ocnerodes Brunner, 1882.

Bolívar (1912) la considera, precedido el nombre específico por un ?, dentro del género Ocneridia Bolívar, 1912 que él crea para englobar los Ocnerodes del norte de África.

De la especie nosotros no tenemos nuevas referencias hasta Harz (1975), quien la considera como sinónima de Acinipe mabillei; esta opinión aparece reflejada en distintas obras posteriores de modo explícito (Presa \& García, 1983; Otte, 1994) e implícito (Presa \& Llorente, 1983; Llorente \& Presa, 1997).

De lo anteriormente expuesto la conclusión obvia sería que $P$. affinis es una especie que, debido a su descripción tan breve, no puede identificarse y además parece ser que el tipo se ha perdido (Otte, 1994: 167 y 176). Por tanto debería ser considerada como especie incierta. 
Esta solución implicaría seguir arrastrando el problema de su ubicación dentro de la fauna ibérica.

Otra opción sería considerarla como especie válida, y suponer que alguna de las restantes especies ibéricas es sinónima suya. Una opción sería la propuesta por Heller et al. (1998), esto es, considerar A. mabillei como su sinónima pues, aplicando el principio de prioridad y teniendo en cuenta la opinión de Harz (1975), sería lo adecuado.

Esta opción, en nuestra opinión, no tiene ninguna razón de ser pues, cuando Bolívar (1878) describe $P$. mabillei, considera $P$. affinis como Pamphaginae inc. sedis; se puede entender, por tanto, que tenía conocimiento de ambas especies y no estableció ninguna relación entre ambas.

En contra de esta opción está, además, la medida $\mathrm{n}^{\mathrm{o}} 11$ de la $4^{\mathrm{a}}$ edición de ICZN, donde se requiere a los autores a no desplazar un nombre que ha sido utilizado como válido al menos por 10 autores en 25 publicaciones durante los últimos 50 años por un sinónimo u homónimo anterior que no ha sido usado válidamente desde 1899. Esta medida se propone con objeto de preservar nombres establecidos y evitar confusiones.

La última opción es seguir la opinión de Harz (1975) y considerar $P$. affinis como sinónimo de $P$. mabillei. Esta opción evitaría todos los problemas que pueden plantear cualquiera de las otras dos. Si desde el punto de vista formal puede tener alguna pega, sí parece la más práctica.

En consecuencia nosotros proponemos que se acepte definitivamente la propuesta de Harz (1975) que es, además, la de uso generalizado.

\section{Eumigus punctatus punctatus (Bolívar, 1902)}

De esta subespecie se capturó una hembra ninfa en Arroyo del Batán, $1.200 \mathrm{~m}$, Bogarra (Albacete) el 30-IV-1995 (A. Sánchez. leg.) en el VI y último estado ninfal, alcanzando el estado adulto entre el 23 y el 24 de mayo. Como adulta sobrevivió hasta el 15 de agosto. Su comportamiento en cautividad es, en general, semejante al ya descrito para otras especies de Pamphagidae. Lo que llama la atención es el modo de producción de sonido que tiene, que representa una novedad para los Pamphagidae ibéricos. Esta hembra comenzó la producción de sonido aproximadamente a los 40 días de ser adulta. Para estridular realiza los siguientes movimientos:

$1^{\circ}$. Se queda quieta, con las antenas rectas y hacia arriba y levanta un poco el pronoto para poder elevar y girar las tegminas. $2^{\circ}$. Levanta las tegminas y las gira unos $90^{\circ}$, poniéndolas verticales en relación al cuerpo y con la banda blanca hacia abajo de tal forma que tanto la cara externa de la tegmina como la interna se ven enteramente. En la cara interna hay dos venas longitudinales muy engrosadas y sobresalientes, en especial la que está en la banda blanca o más próxima a ella.

$3^{\circ}$. Las tegminas empiezan a batir o aletear de izquierda a derecha y viceversa con bastante rapidez y frotándolas a su vez contra el borde costal de las alas posteriores que aparece como una costilla engrosada de sólo un milímetro de longitud. Cuando la hembra estridula, toda ella vibra, pero bastante menos que en otros casos descritos (Llorente et al., 1995; García et al., 1996).

Este método de estridulación fue observado y descrito con el nombre inglés de "flapping" - batir o aletear - por Johnsen (1972) al estudiar los panfágidos Acinipe crassicornis (Bolívar, 1907) y Glauia durieui (Bolívar, 1878), no habiendo sido descrito de nuevo hasta ahora.

Después de haber realizado varios cantos con el método anteriormente descrito, una de las tegminas quedaba horizontal y seguidamente golpeaba contra la rodilla del segundo par de patas, utilizando el método de "tapping" — tamborileo- Este hecho se repitió varias veces, pero siempre con una sola de las tegminas; en algunos casos la misma tegmina empezaba su estridulación aleteando y la acababa tamborileando. El sonido producido es más corto y mucho más suave y menos perceptible que el de E. punctatus templadoi Llorente y Presa, 1996; desgraciadamente un fallo mecánico impidió el registro del mismo. La hembra estuvo produciendo sonido desde el 3 de julio hasta el 11 del mismo mes.

En el mismo contenedor en que se mantuvo esta hembra había un macho de Kurtharzia nugatoria (Navás, 1909) que intentó realizar con ella repetidamente la cópula. En un principio la hembra trataba de rechazarle con las patas posteriores, al no conseguirlo seguía su actividad normal desplazándose por la jaula con el macho encima. En ningún momento se observaron cópulas.

\section{Kurtharzia nugatoria (Navás, 1909)}

Navás describió esta especie sobre un macho recolectado en Puertos de Beceite y ejemplares de Segorbe (Pau leg.); estos ejemplares habían sido citados por Pau en Navás (1906) como Pamphagus cucullatus Bolívar. Dicho macho y una hembra de 
Segorbe parecen ser los únicos ejemplares que quedan de la serie tipo y se conservan en las colecciones del MNCN de Madrid. Designamos el macho como lectotipo por ser el sexo más característico, y también por llevar etiqueta de "Typus", probablemente del mismo Navás. La primera etiqueta lleva escrito ¿con letra del autor?: "Pamphagus nugatorius Nav. Beceite (Teruel) $10 \mathrm{Ag}^{\mathrm{o}}$ 1908"; la segunda, en papel rosa decolorado, "TYPUS" y la tercera, con letra de I. Bolívar: "Eumigus Bol. nugatorius Nav. Esp.”. La hembra, designada como paralectotipo, lleva sobre la primera etiqueta escrito a mano: "Pau. Segorbe" y en la segunda, con letra de I. Bolívar: "Eumigus nugatorius Nav.".

\section{AGRADECIMIENTOS}

Queremos manifestar nuestro agradecimiento al Dr. R Gómez, de la ETSI Agrónomos de la Universidad de CastillaLa Mancha por la ayuda que nos ha prestado para la realización de este trabajo.

\section{Referencias}

Barranco, P., Baena, M. \& Pascual, F., 1996. Los Orthoptera de la provincia de Córdoba (España). Zoologica baetica, 7: 61-77.

Bolívar, I., 1876. Sinopsis de los Ortópteros de España y Portugal. Anales de la Sociedad Española de Historia Natural, 5: 284-291; 364-366.

Bolívar, I., 1878. Analecta Orthopterologica. Anales de la Sociedad Española de Historia Natural, 7: 423470.

Bolívar, I., 1912. Los Panfaginos paleárticos. Trabajos del Museo de Ciencias Naturales, Madrid, 6: 1-32.

BrunNer, C., 1882. Prodromus der Europäischen Orthopteren. Leipzig. 466 pp.

Cazurro, M., 1888. Enumeración de los Ortópteros de España y Portugal. Anales de la Sociedad Española de Historia Natural, 17: 435-513.

Clemente, M.E., García, M.D. \& Presa, J.J., 1990. Datos sobre el comportamiento en cautividad de Navasius sulcatus Bolívar, 1912 (Orthoptera, Pamphagidae). Zoologica baetica, 1: 33-42.

García, M.D., Clemente, M.E., Llorente, V. \& Presa, J.J., 1996. Datos sobre el comportamiento de Eumigus cucullatus (Bolívar, 1878), Eumigus monticola (Rambur, 1838) y Ocnerodes fallaciosus Bolívar, 1912 (Orthoptera: Pamphagidae): manifestaciones acústicas. Boletín de la Asociación española de Entomología, 20(1-2): 161-171.

GARcíA, M.D. \& PresA, J.J., 1985. Observaciones sobre el comportamiento en cautividad de Eumigus cucullatus (Bolívar, 1878) (Orth., Pamphagidae).
Boletín de la Asociación española de Entomología, 9: 175-181.

HARZ, K., 1975. The Orthoptera of Europe. II. Vol. 11. Series Entomologica. The Hague. 939 pp.

Heller, K.-G., Korsunovskaya, O., Ragge, D.R., Vedenina, V., Willemse, F., Zhantiev, R.D. \& FrantSEVICH, L., 1998. Check-list of European Orthoptera. Articulata, b.7: 1-61.

Hernández, A., Clemente, M.E., García, M.D. \& PRESA, J.J., 1998. Inventario y dinámica poblacional de los ortopteroides (Orthoptera, Blattodea, Mantodea y Phasmoptera) del parque natural del "Carrascal de la Font Roja" (Alicante, E. España). Zoologica baetica, 9: 185-204.

Johnsen, P., 1972. Alar-notal and tegmino-alar sound production in the African Glauia and Acinipe (Acridoidea: Pamphagidae), with notes on other behaviour. Natura Jutlandica, 16: 61-80.

KIRBY, W.F., 1910. A synonymic catalogue of the Orthoptera. III. Orthoptera Saltatoria. Part. II. (Locustidae vel Acridiidae). British Museum (Natural History). London. 674 pp.

Llorente, V., García, M.D. \& Presa, J.J., 1995. Datos sobre el comportamiento en cautividad de Eumigus punctatus templadoi Llorente y Presa, 1986 (Orthoptera, Pamphagidae). Zoologica baetica, 6: 23-32.

Llorente, V. \& Presa, J.J., 1997. Los Pamphagidae de la Península Ibérica (Insecta: Orthoptera: Caelifera). Universidad de Murcia. Servicio de Publicaciones. 248 pp.

NAVÁs, L., 1906. Notas zoológicas XII. Novedades zoológicas de Aragón. Boletín de la Sociedad Aragonesa de Ciencias Naturales, 5: 201-203.

OTTE, D., 1994. Orthoptera species file e. Grasshoppers [Acridomorpha]. B-. Pamphagoidea. Publications on Orthopteran Diversity. The Orthopterists' Society and The Academy of Natural Sciences of Philadelphia. $241 \mathrm{pp}$.

PRESA, J.J. \& GARCÍA, M.D., 1983. Inventario preliminar de los Pamphagidae paleárticos (Orthoptera). Anales de la Universidad de Murcia, Ciencias, 42(1-4): 342.

Presa, J.J. \& Llorente, V., 1983. Los Pamphagidae de la Península Ibérica. I. Gén. Prionotropis Fieb. y Acinipe Ramb. Eos, [1982], 58: 271-302.

Recibido, el 14-I-2000 Aceptado, el 20-VII-2000 Publicado, el 27-XII-2000 